Review Article

\title{
Parent-Infant Interaction during the First Year of Life in Infants at High Risk for Cerebral Palsy: A Systematic Review of the Literature
}

\author{
F. Festante $\mathbb{D}^{1},{ }^{1}$ C. Antonelli, ${ }^{1}$ O. Chorna ${ }^{\mathbb{D}},{ }^{1}$ G. Corsi ${ }^{D},{ }^{1}$ and A. Guzzetta ${ }^{1,}{ }^{1,2}$ \\ ${ }^{1}$ Department of Developmental Neuroscience, IRCCS Fondazione Stella Maris, Pisa, Italy \\ ${ }^{2}$ Department of Clinical and Experimental Medicine, University of Pisa, Pisa, Italy \\ Correspondence should be addressed to A. Guzzetta; a.guzzetta@fsm.unipi.it
}

Received 17 August 2018; Revised 18 January 2019; Accepted 4 February 2019; Published 15 April 2019

Academic Editor: Francesca Foti

Copyright (c) 2019 F. Festante et al. This is an open access article distributed under the Creative Commons Attribution License, which permits unrestricted use, distribution, and reproduction in any medium, provided the original work is properly cited.

\begin{abstract}
Introduction. Perinatal adverse events put neonates at high risk for short and long-term disabilities, including cerebral palsy (CP). The most recent guidelines about early intervention in infants with brain damage have emphasized the importance of family involvement from the very first phases of development. Early parent-infant interactions are pivotal in promoting infant cognitive and social developmental trajectories. However, little is known about the extent to which severe adverse perinatal events can affect the quality of early parent-infant interactions. Patients and Methods. We systematically searched five databases (PubMed, PsycINFO, EMBASE, CINAHL, and Cochrane Library) for the publications assessing parent-infant interactions in infants at high neurological risk within 1 year of age. Articles were selected if they involved direct comparison between high-risk populations and healthy controls or low-risk populations, and if quantitative or semiquantitative tools were used to assess the parent-infant interaction. Measures of parent-infant interaction included infant interactive behaviors, parental interactive behaviors, and dyadic interactive patterns. Results. The search yielded 18 publications that met the inclusion criteria. The articles represent a high level of heterogeneity in terms of infant neurological risk, infant age, and tools assessing interactive behaviors. Both infant and maternal behaviors within the investigated interactive exchanges were reported to be compromised, leading to subsequent overall impairment of the dyadic patterns. Conclusion. While the studies reviewed here provide general and important information, the review did not yield a clear picture of early dyadic interactions in high-risk infant populations. Further observational studies are warranted in order to provide a more accurate knowledge of the early dyadic exchanges between infants at high neurological risk and their parents, as they might provide a critical opportunity for early family centered habilitative interventions.
\end{abstract}

\section{Introduction}

The role of parent-infant interaction during early development has been studied extensively in the past decades. Newborn's brain is known to be prone to interactive exchanges at birth or even before $[1,2]$. Neonatal imitative processes, occurring from the very first hours of life [2], represent the first signs of reciprocity between parents and infants that, within the first months of life, evolve towards actual protoconversations, characterized by reciprocal multimodal exchanges and rhythmic vocal, facial, and gesture imitations [3-5]. Murray and colleagues have recently suggested the existence of a functional architecture of mother-infant engagements, active from the very first weeks of life and apt to support the development of infant intersubjective skills [6]. Authors reported that the occurrence of mirroring or marking maternal responses to infant social expressions predicts the increase of such infant behaviors over time. More importantly, they stressed the importance of contingency more than frequency of maternal responses, thus suggesting that infants are able to capitalize on relatively limited exposure to specific parental behaviors, already at very early developmental stages.

Primary dyadic interactions support infants' cognitive, motor, and social skills maturation $[7,8]$. Studies in typically 
developing infants have widely demonstrated that the quality of early dyadic interactions can strongly influence later infants' developmental outcomes [9-12]. For instance, Feldman and Greenbaum [10] reported that maternal affective attunement and dyadic synchrony within the interaction of 3 -month-old infants and their mothers were predictive of infants' quality of play, verbal IQ, and regulation capacity at 2 years of age. The contingency within interactive exchanges at 3 months of age has been reported to be a precursor of infants' attachment style at 1 year [13], while maternal sensitivity to infantdistresshasbeendescribedasapredictorofasecureattachment [14].Accordingly, studiesfocusingonclinicalpopulations showed that the occurrence of either parents adverse conditions (e.g., maternal depression, anxiety or early traumatic experiences, and poor socioeconomic family) or infant pathological conditions (e.g., preterm birth, autism, and cleft lip palate) can beassociated with poorinfantaffective, social, and cognitiveoutcomes, likely due, at least partly, to a disruption of the quality of early dyadic interactions [7, 15-17].

So far, little attention has been given to the quality of early dyadic interaction in infants at high risk of neuromotor disabilities, and more specifically of cerebral palsy (CP), as studies on term infants with brain damage are very scarce, while the majority of the studies on preterm infants have focused on low-risk prematurity $[18,19]$.

$\mathrm{CP}$ is the most common physical disability in childhood [20]. Despite the progressive improvements in perinatal and neonatal care, extreme prematurity as well as perinatal insults are still associated with major neonatal morbidities with longterm sequelae such as neurodevelopmental delay, neurosensory disorders, and cerebral palsy [21-25]. More specifically, in populations of very or extremely premature infants or of full-term infants with a history of perinatal asphyxia, the prevalence rate of $\mathrm{CP}$ is still consistently above $10 \%$ in high-income countries [26-30]. Scientific evidence is rapidly growing in support of the importance of an early diagnosis of $\mathrm{CP}$ for the improvement of long-term outcomes [31]. This is essential for a prompt referral to early intervention programs aimed at promoting and maximizing neuroplasticity, minimizing further medical complications [31], and providing emotional support for parents [32-34].

The most recent guidelines about early intervention in infants with brain damage have greatly emphasized the importance of family involvement from the very first phases of development [35]. Indeed, review studies in infants at very high risk of $\mathrm{CP}$ indicate that early interventions focusing on parents' empowerment and supporting early parent-infant relationships may have a greater impact on later cognitive and neuromotor outcomes compared to those with an exclusive focus on infant functional impairment $[36,37]$. A deep knowledge of general and detailed aspects of parent-infant interaction in populations at high risk of $\mathrm{CP}$ would be therefore essential to inform new strategies for early clinical support in both infants and parents. Unfortunately very little is known about how the parent-infant dyad is affected by the occurrence of severe perinatal events. In order to contribute to bridging this knowledge gap, we systematically reviewed the existing literature on early dyadic interactions between parents and infants at high risk of neurological impairments.
The main objective of this paper was to review the current knowledge on the influence of severe adverse perinatal events on the quality of early parent-infant interaction, focusing on infant behavior, parental behavior, and dyadic interactive patterns. We specifically focused on papers evaluating dyadic interactions occurring within the first year of life, as the optimal time window of the emergence and early development of infant and parental interactive patterns.

\section{Methods}

2.1. Literature Search and Selection of Studies. A systematic literature search was performed in February 2018, through the following electronic databases: PubMed/MEDLINE, PsycINFO, EMBASE (OVID), CINAHL, and Cochrane Library. No publication date limits were applied to the searches.

The following search strategy, including both MeSH headings and keywords, was used: (Parent-child relations (Mesh) OR Mother-Child OR Father-Child OR Parent-Child OR Mother-Infant OR Father-infant OR Parent-infant) AND (Interaction* OR Relation* OR Attachment* OR Bond* OR Intersubjectiv* OR Transact*) AND (Brain injuries (Mesh) OR Brain damage* OR Brain Injury* OR Brain lesion* OR Brain malformation OR Asphyxia OR Hypoxia OR Ischemia OR Encephalopathy OR Hypoxic Ischemic Encephalopathy OR Cerebral stroke OR Leukomalacia OR Hemorrhage OR Haemorrhage OR High-risk) AND (Infant (Mesh) OR Infant OR Newborn* OR Neonate* OR baby OR Preterm OR Premature).

The list of records was first checked for duplicates using EndNote (EndNote X8.2, bld 13302). Subsequently, two authors (FF and CA) independently reviewed the remaining records for suitability by title and abstract. Finally, full-text articles addressing the topic of interest were screened in order to exclude those not meeting inclusion criteria. Secondary searches involved checking of publication reference lists and manual searches of relevant journals.

Agreement for articles inclusion was reached upon discussion between authors (FF, CA, and AG).

2.2. Inclusion Criteria. Article selection was restricted to peer-reviewed research articles published in English and to human studies. Articles were selected if they met all of the following criteria: (1) the study involved direct comparison between at least one population of infants at high risk for neurological impairment and either healthy controls or low-risk populations, (2) mother-infant and/or father-infant and/or both parents-infant interactions were assessed, (3) quantitative or semiquantitative tools were used to assess the interaction, (4) the study included assessments within the first year of the infant's life.

High risk for neurological impairment was defined by one or more of the following conditions: gestational age (GA) at birth under 30 weeks, birth weight (BW) below $1500 \mathrm{~g}$, perinatal asphyxia or hypoxic ischemic encephalopathy, cerebral stroke, periventricular leukomalacia, severe intraventricular hemorrhage (grade III or IV), or any type of documented brain damage occurring within the first month of life. Populations were defined as at high risk for 
neurological impairments if at least $50 \%$ of the participants met the above criteria.

No limitations for article inclusion were applied to parent-infant interaction assessment methods, which could include feeding sessions, face-to-face interactions, and free or structured play sessions, either toy-centered or non-toycentered. Similarly, early interaction scoring modalities including scoring scales, manuals, or checklists were included provided that a clear description of the analyzed parental and/or infant interactive dimensions (e.g., maternal intrusiveness, infant engagement, and dyadic synchrony) were reported.

2.3. Data Extraction. Descriptive information of all included articles was systematically extracted and gathered in an electronic database. These included authors, year, study design, sample size, inclusion and exclusion criteria for clinical and/or control samples, age of infants at the time/s of the parent-infant interaction assessment, assessment methods (e.g., place, duration), scoring modalities (e.g., behavioral annotation), and main findings relative to the early parent-infant interaction. Additional parental, neonatal, or developmental outcome measures and any other results relevant to the current report were scored and gathered, if reported. The quality of the included studies was assessed by using the National Heart, Lung, and Blood Institute (NHLBI) Quality Assessment Tool for Case-Control Studies [38], which was chosen based on the study design of the included articles. Two authors (OC and FF) independently evaluated the items of the tool as "yes," "no," "not applicable," "cannot determine," or "not reported." The comprehensive evaluation of all items was then used to rate the global quality of each study as "good," "fair," or "poor."

2.4. Measures of Parent-Infant Interaction. Measures of parent-infant interaction were grouped into three categories. (i) Infant interactive behaviors included all behaviors originated by the infant as either initiations or responses within the assessed interactions, such as activity (e.g., movements, vocalizations, or expressive language) and engagement (e.g., facial expressions or eye contact). (ii) Parental interactive behaviors included all dimensions originated by the parent as either initiations or responses within the assessed interactions, such as sensitivity, vigilance, intrusiveness, and emotional involvement (e.g., kinesthetic or proximal stimulation, smiling, vocalizations, time spent looking at infant, and proximity to infant). (iii) Dyadic interactive patterns included all behaviors of the dyad observed as a single entity within the assessed interactions, such as synchrony, reciprocity, and coregulation (e.g., timing, rhythmicity, and fluency of interactive exchanges).

\section{Results}

The flow chart in Figure 1 summarizes the whole selection process and exclusion criteria at each selection step. Database and secondary searches yielded 2910 articles. After duplicates removal, titles and abstracts of 2673 articles were screened. Then, 82 full-text articles were scored, of which 18 met all predetermined inclusion criteria and were included in the present review. Overall, the quality of the studies was evaluated as fair or good with the exception of one paper whose quality was rated as poor. Details of the studies, including the quality rating, are summarized in Table 1.

3.1. Level of Neurological Risk of the Study Populations. All studies included preterm populations, while few of them involved mixed populations of preterm and full-term infants at high risk of neurological impairments. The severity of infant risk status was varied among the selected articles. In 8 articles, the high-risk population presented intraventricular hemorrhage (grades III-IV), periventricular leukomalacia, severe perinatal asphyxia, seizures, meningitis, or other severe medical conditions $[39,45,46,49-51,55,58]$, while in the remaining studies, the high-risk population included infants on the basis of intrauterine growth retardation (IUGR), birth weight, or prematurity with or without mild or moderate medical complications $[41,42,53,60,62,64,65,67,69,70]$.

Seven of the 18 studies explicitly excluded infants with severe brain lesions or perinatal asphyxia from their highrisk samples [50, 51, 60, 62, 67, 69, 70]; however, the resulting populations still met at least one of our inclusion criteria for high neurological risk (i.e., BW or GA) and were therefore retained in the current review.

3.2. Parent-Infant Interaction Assessment Modalities. The time duration for observation of early parent-infant interaction was also heterogeneous, varying from few minutes long video-recorded sessions (from 3 to 20 minutes) [39, 49, 51, $53,58,62,64,67,69]$ to much longer (up to 120 minutes) live observations $[41,42,46,50]$. A wide variety of parent-infant interaction coding modalities was used, including microanalytic coding systems $[42,45,49,58,60,70]$, rating scales [46, $53,55,62,64,65,67,69]$, and scoring checklists $[39,41,51]$. Only one study used a mixed coding system which included both microanalytic and global rating scores [50]. Assessment details are summarized in Table 2.

The timing at which parent-infant interaction assessments were performed was equally distributed over the first year of the infant life, with about half of the included studies collecting data within the first semester of life $[39,42,51,53,58,60,62,64$, 67, 69] and half focusing on older infants, from 6 to 12 -month-old infants [41, 45, 46, 49, 50, 55, 65, 70]. Most of the studies involved a cross-sectional design $[39,41,45,46$, $53,58,60,64,67,69,70]$, while few of them implemented a longitudinal design, with data collection at multiple time points $[42,50,51,65]$. Three studies $[49,55,62]$ evaluated motherinfant interaction over a longer period of time, which was beyond the first year of life. For these studies, however, only assessments that occurred within the first year of life were considered in the present review.

Finally, all studies focused on mother-infant interaction. Feldman [64] was the only study that, in addition to the mother-infant interaction, also included father-infant and triadic early interactions. The authors found no differences between father-infant and mother-infant interactions; 


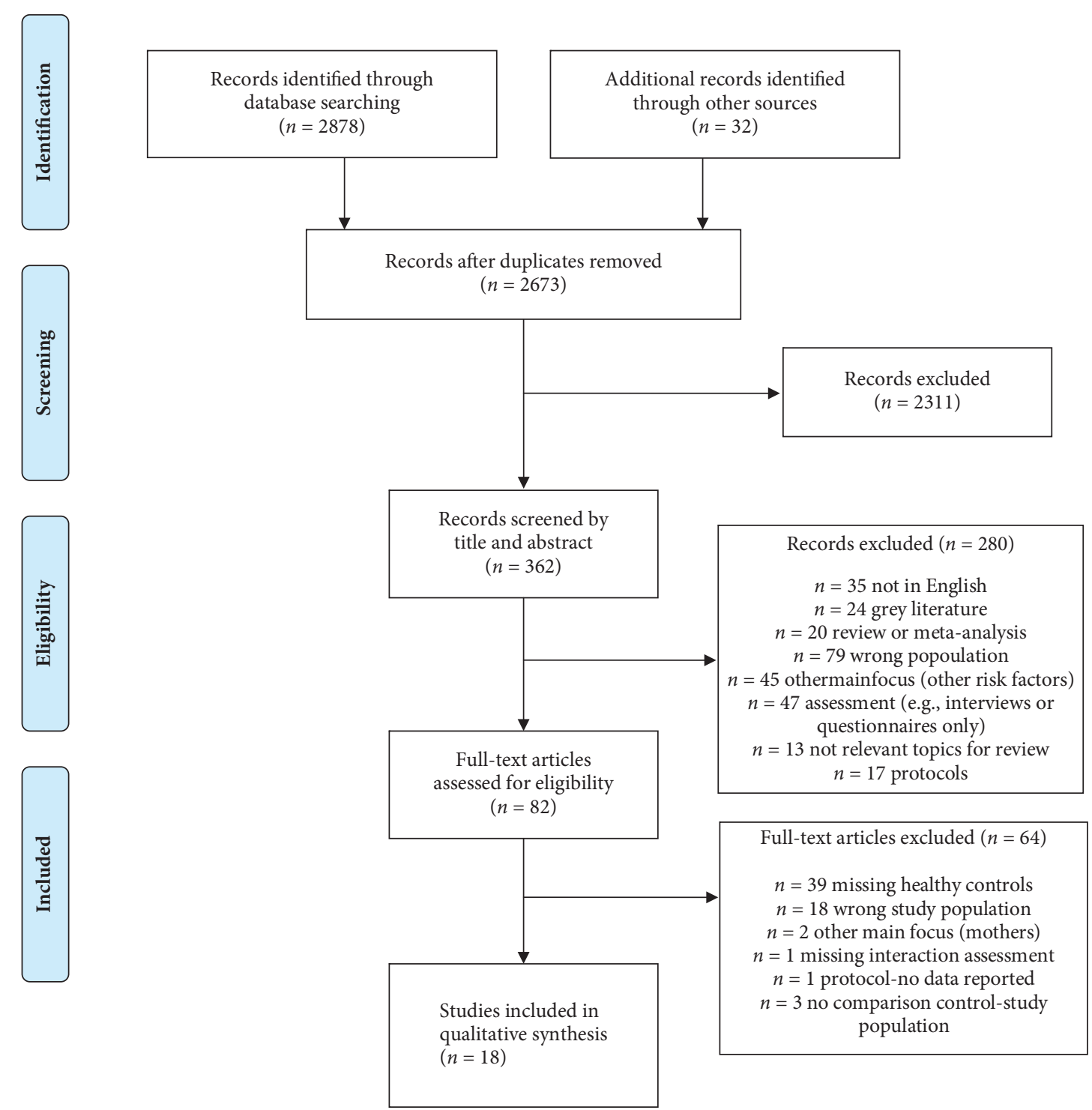

FIGURE 1: PRISMA flow chart. Flow chart of literature search and study selection.

therefore, we abstain from discussing this topic further in this review.

3.3. Comparison of Interactive Patterns between High-Risk and Non-High-Risk Dyads prior to 6 Months. Overall, all of the included studies described compromised dyadic interactive patterns between mothers and infants who experienced adverse perinatal events, compared to those occurring within control populations. While most of the studies focused on interactive behaviors considering mother and infant as discrete interacting units, few studies analyzed the quality of early interactive exchanges from a dyadic perspective. Due to the variety of the interaction assessments and the scoring modalities utilized within the included studies, a wide range of parental and infant dimensions were analyzed. However, a categorization of common terms used within the selected publications is included for our reporting purposes (see bold text in the following section). Therefore, below, we report the findings in three sections, divided into groups of behaviors or behavioral dimensions.

3.3.1. Infant Interactive Behavior. Within the first semester of life (corrected age for prematurity), infants at high risk were generally described as less active, less engaged in the interaction, and more fretful than controls. Minde and colleagues [42] reported that preterm infants were less alert and focused, as revealed by the fact that they spent less time with their eyes open during feeding sessions at one month of age, although they became more physically active by the third month of life. Davis et al. [53] described preterm infants at risk as less responsive to their caregivers compared to typically developing infants, during feeding. Schmücker et al. [58] and Feldman [64], instead, found that high-risk preterm infants were less optimally engaged in dyadic interaction with their mothers, as they showed less facial expressions and more negative engagement cues, 


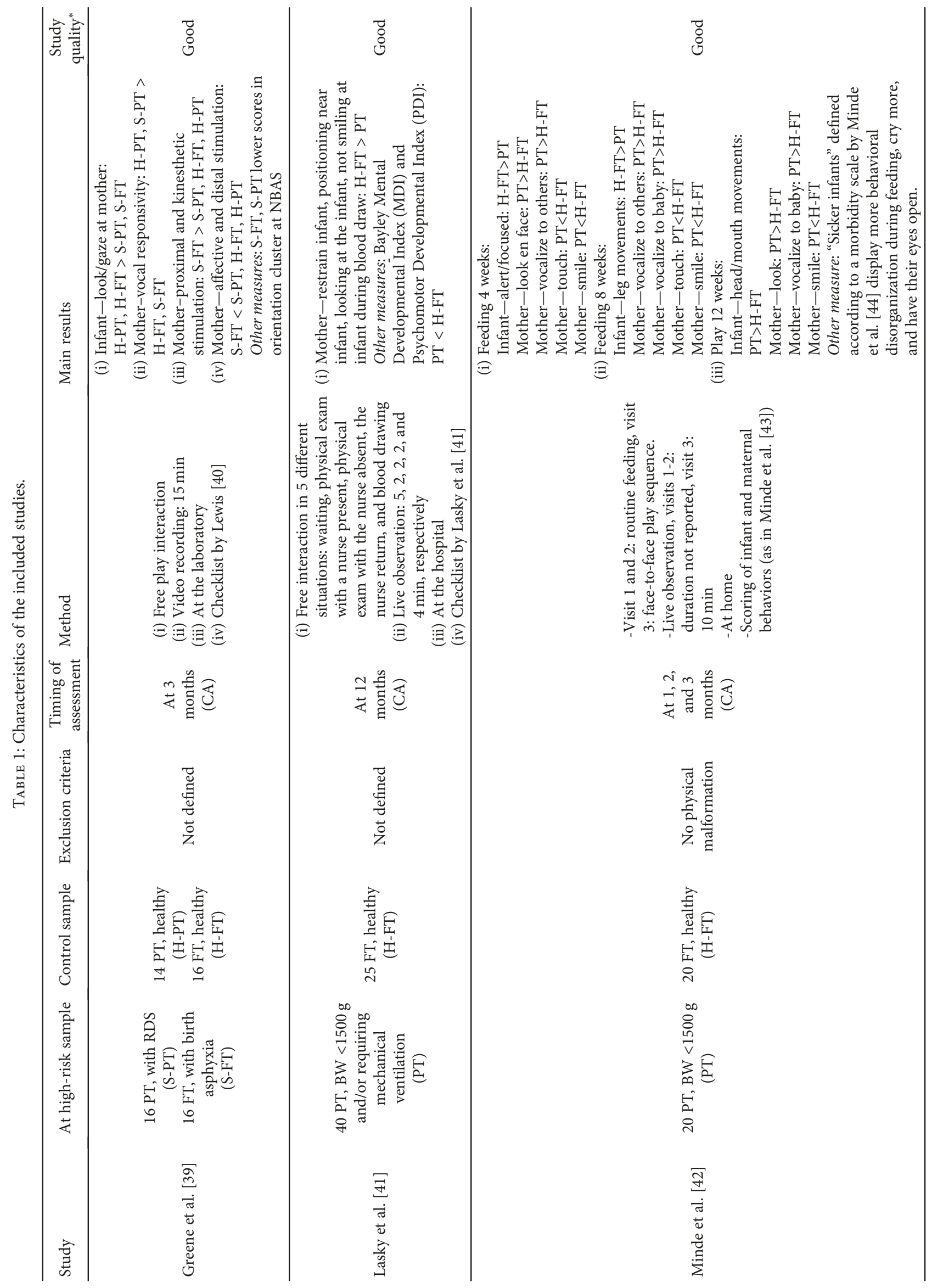




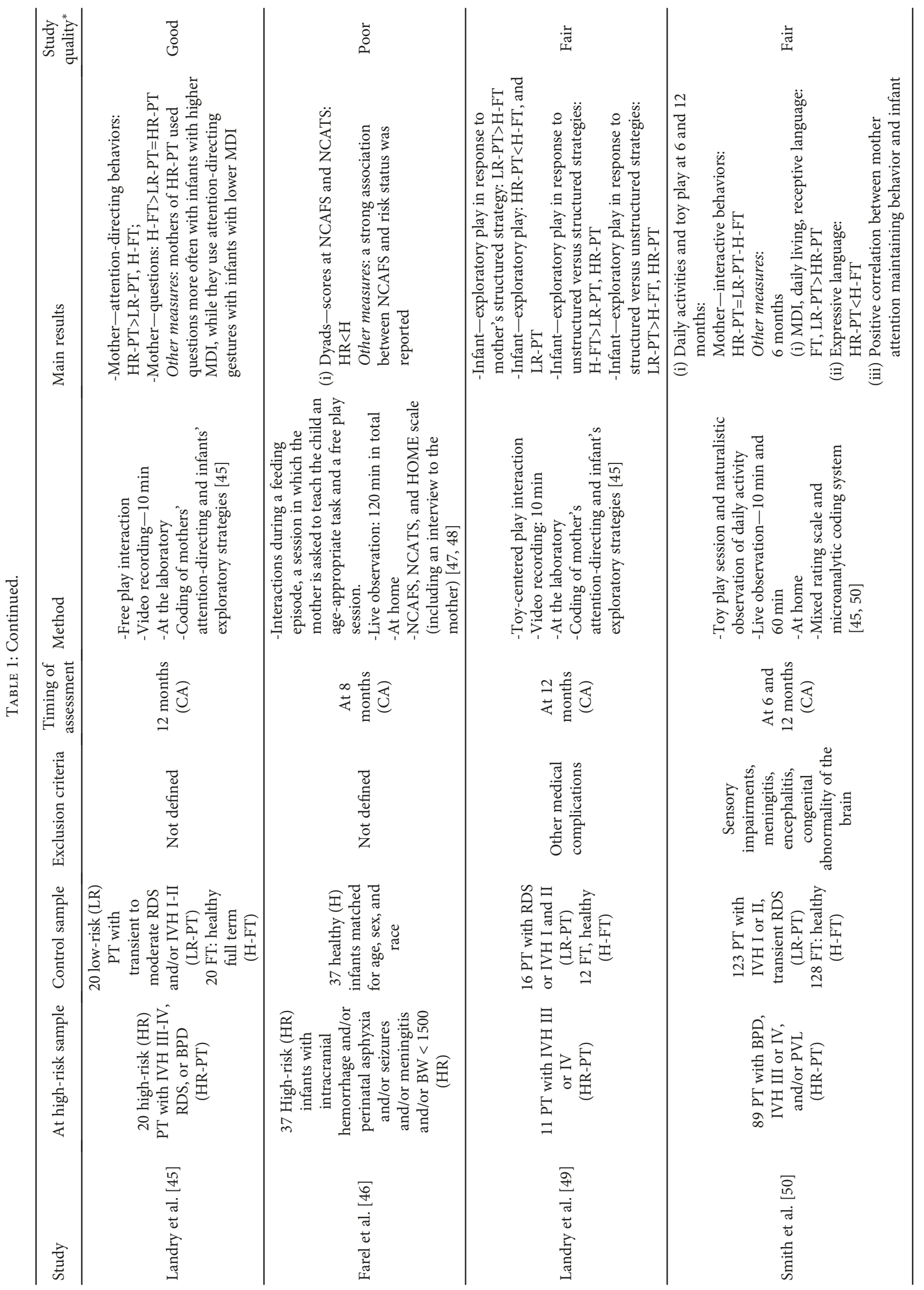




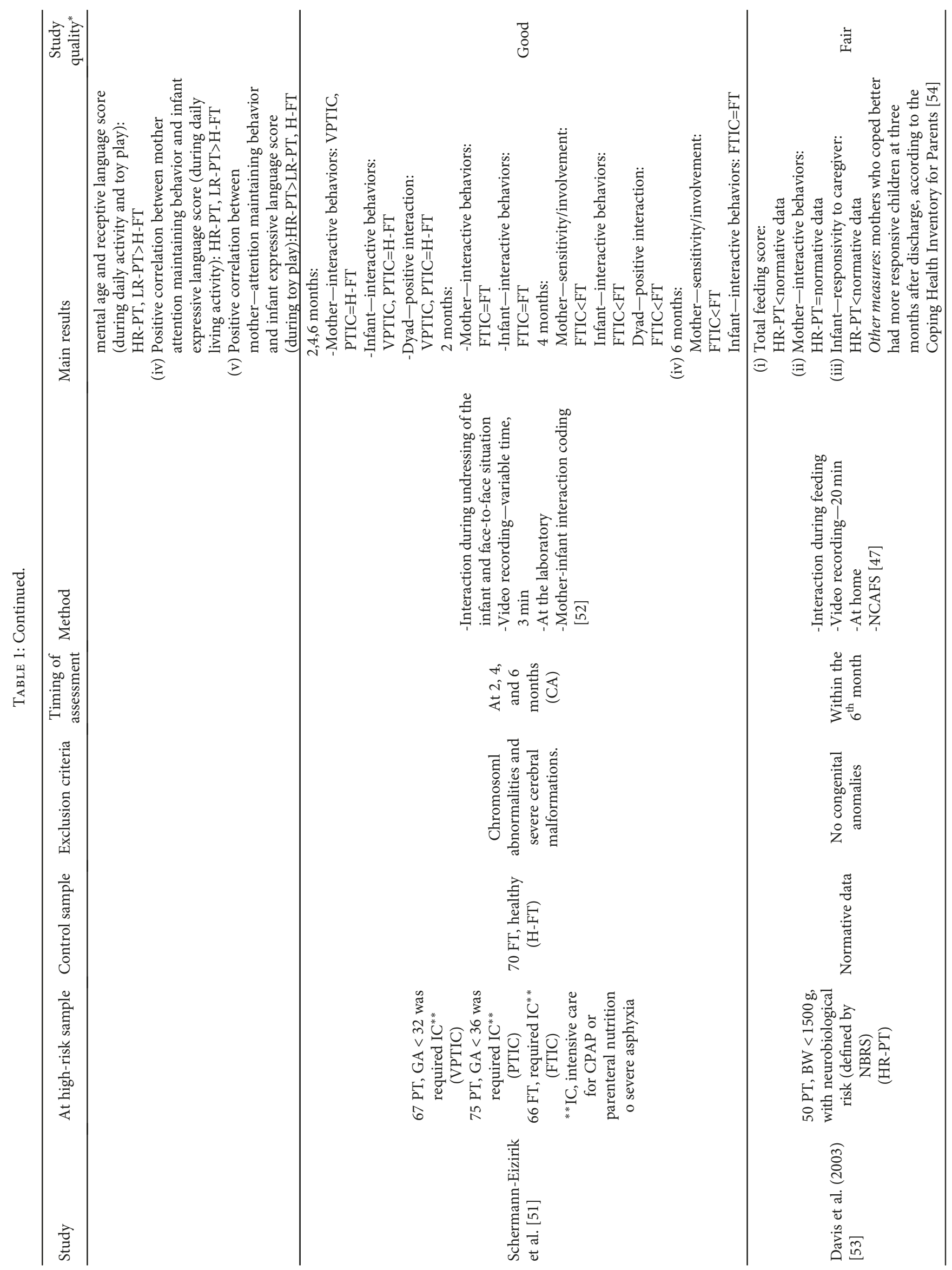




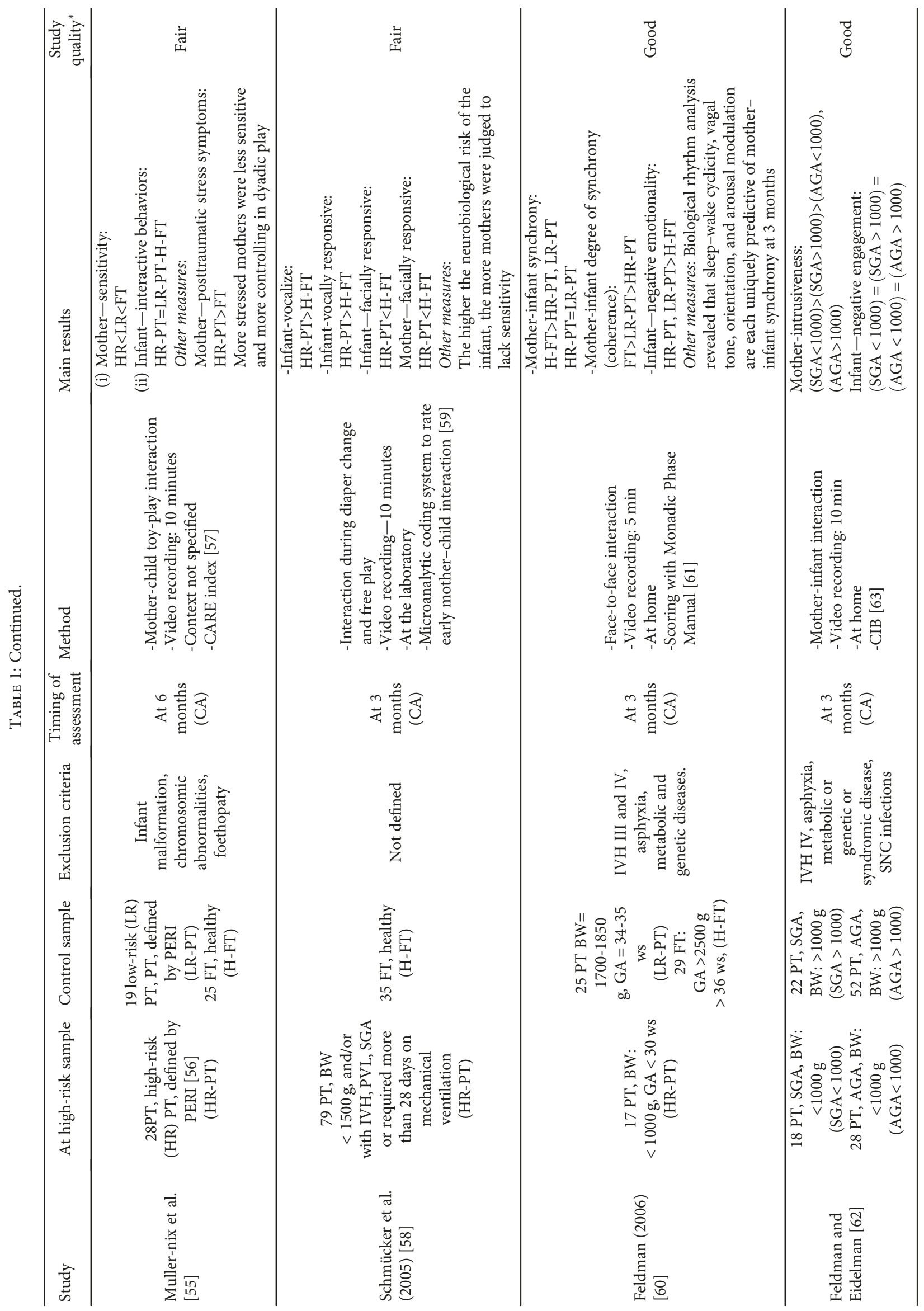




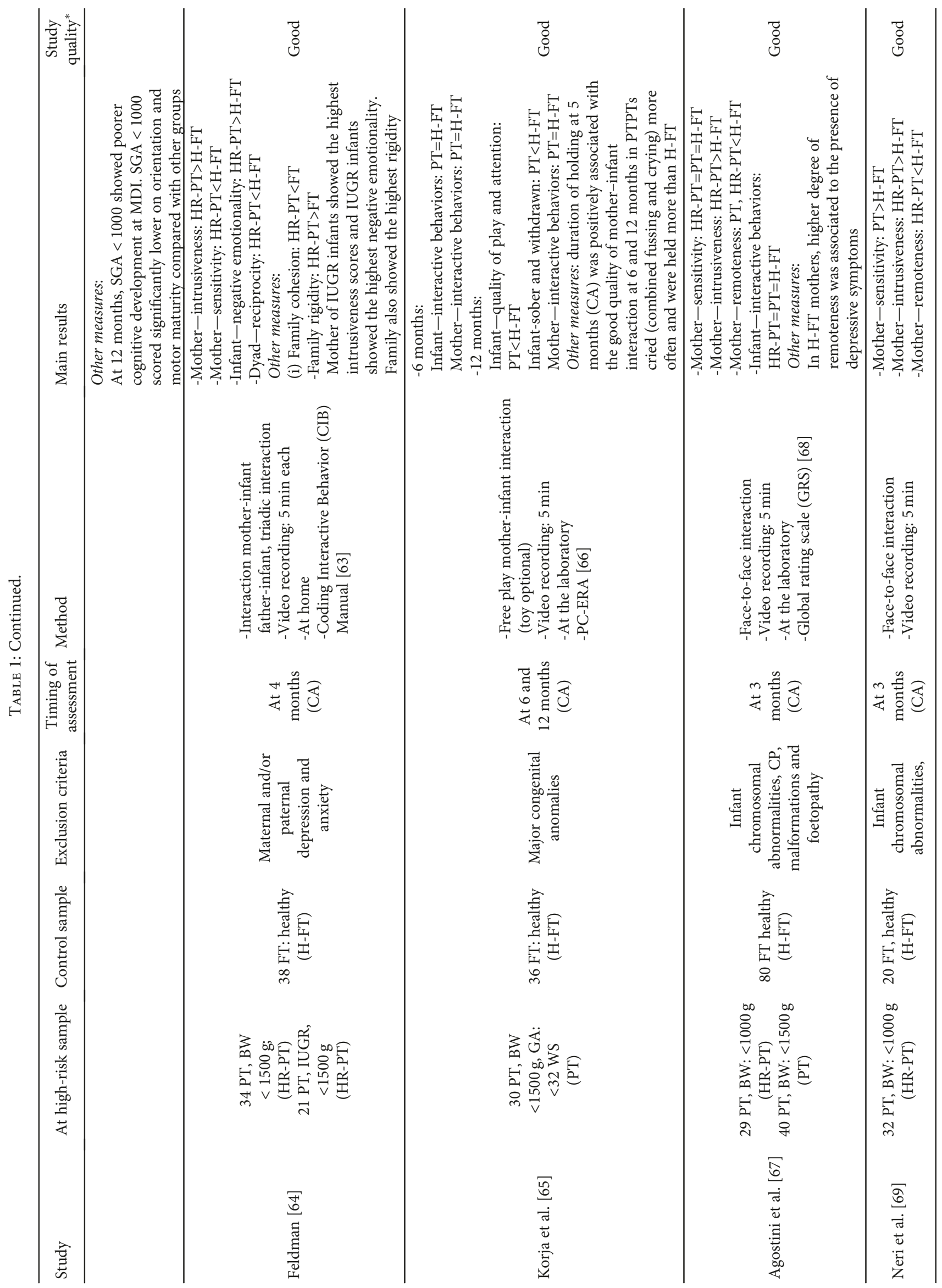




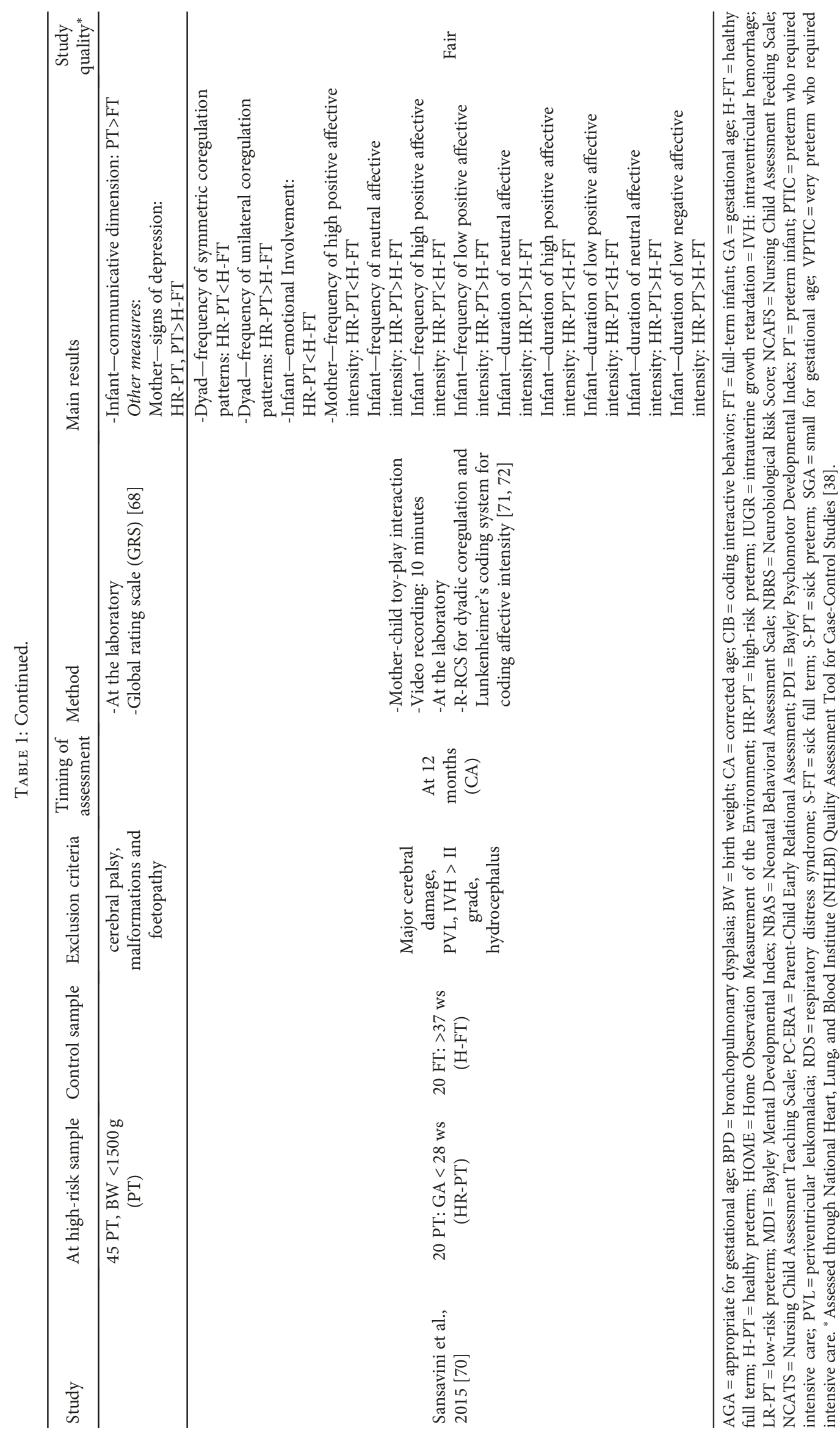


TABLE 2: Assessment and scoring scales used in the studies.

\begin{tabular}{lll}
\hline $\begin{array}{l}\text { Assessment and scoring } \\
\text { scales }\end{array}$ & Description & Reference Studies
\end{tabular}

Global scales

NCAST Feeding (NCAFS) and Teaching (NCAST)

PCI Scales

Coding Interactive Behavior (CIB)

Parent-Child Early Relational Assessment (PC-ERA)

CARE-Index

HOME
Assessment of the quality of mother-infant interaction. Maternal behavior is rated on 4 dimensions: sensitivity, intrusiveness, remoteness, and signs of depression. Infant behavior is rated on 3 dimensions: communicative, inert, and distressed. One dimension assesses the quality of the overall interaction between mother and infant. A 5-point Likert-type scale is used to rate each dimension, with 1 being the poorest and 5 being the optimal rating.

The NCAST-PCI evaluates 149 items related to maternal and infant behaviors. It comprises two scales: NCAFS and NCATS. Infant and parent items are coded as yes or no; items are then added to provide a total score. Each scale includes 4 subscales, measuring maternal behaviors and 2 subscales, measuring infant's behaviors.

Maternal subscales are sensitivity to cues, responsivity to child's distress, social-emotional growth fostering, and cognitive growth fostering. Infant subscales are clarity of cues and responsiveness to parent.

Global rating system of parent-child interaction in different play or interaction situations, including 42 codes: 21 for parents, 16 for infants, and 5 for dyads. Each score is rated with a Likert-type scale, where 1 corresponds to the poorest and 5 to the optimal rating. Five composite scales are included: maternal sensitivity, maternal intrusiveness, child's social involvement, and dyadic reciprocity.

Semistructured assessment to evaluate affective and behavioral quality of parent-infant interaction during 4 situations: feeding, administration of a structured task, free play, and a separation-reunion task. Three parental subscales (29 items) are coded: positive affective involvement and verbalization, negative affect and behaviors, and intrusiveness, insensitivity, and inconsistency. Three Infant subscales (28 items) are coded: positive affect, social and communicative competence; quality of play, interest and attentional skills; dysregulation and irritability. Two dyadic subscales (8 items) are coded: mutual enjoyment and reciprocity, tension and disorganization. A 5-point Likert-type scale is used to rate each item.

Assessment of the quality of adult-infant interaction. Three adult behaviors are scored: sensitivity, control, and unresponsiveness.

Four infant behaviors are scored: cooperativeness, compulsiveness, difficultness, and passivity. The scores range from 0 to 14 , with 0 score being the worst score.

Inventory designed to identify the presence of risk for developmental delay due to lacking of appropriate quantity and quality of stimulation from home environment. Forty-five binary items, organized in six subscales, are scored using a combination of semistructured mother interview relative to children routine activities, observation of mother-infant interaction during play and interview and assessment of kinds of play materials available to the child. Six subscales are coded: emotional and verbal maternal responsivity,
Gunning et al.

Murray et al. [15, 73]

Agostini et al. [67];

Neri et al. [69]

Barnard et al. [47]

Davis et al. [53]

Farel et al. [46]

Feldman [63]

Feldman and

Eidelman [62];

Feldman [64]

Clark [66]

Korja et al. [65]

http://www. patcrittenden.com, Crittenden 1979-2004 [57]

Bradley and Cadwell [48]

Farel et al., [46]
Muller-nix et al. [55] 
TABLE 2: Continued.

\begin{tabular}{l}
$\begin{array}{l}\text { Assessment and scoring } \\
\text { scales }\end{array}$ \\
\hline $\begin{array}{r}\text { maternal avoidance of restriction and punishment, } \\
\text { maternal involvement with the child, organization of the } \\
\text { environment, provision of appropriate play materials, } \\
\text { and variety in daily stimulation. }\end{array}$ \\
Microanalytic
\end{tabular}

Microanalytic

Coding system of Minde (1980)

Microanalytic coding system to rate early mother-child Interaction by Jorg (1994)

Coding system of Landry (1986)

Monadic Phase Manual

Revised relational coding system (R-RCS)

Lunknenheimer's coding system
Microanalytic system recording the occurrence of the 10 maternal and 11 infant behaviors. Infant behaviors: arm, head, leg, hand to mouth; eyes open; scan; grimace; cry; vocalize; smile; yawn.

Maternal behaviors: look; look en face; verbalize to baby and to others; instrumental and noninstrumental touch; hold; feed; smile; standing further than 1 meter away from the baby.

Microanalytic system which rates interactive behaviors at fixed time intervals of 1,15 , and 30 seconds. In particular, maternal behaviors rated per second are direction of gaze, vocalization, facial expression, content of interaction, and proximity; infant behaviors rated per second are direction

of gaze, vocalization, and facial expression; joint mother-child behaviors rated every 15-30 seconds are appropriateness of stimulation, maternal responsiveness, and child responsiveness.

This coding system is based on the recording of the occurrence of mothers' attention-directing strategies and infants' responses.

The variables scored are maternal attention-directing attempt, verbal technique-question, verbal

technique-imperative, verbal technique-attention verbs, nonverbal techniques attention directing-gesture, nonverbal techniques attention directing-demonstrate, nonverbal techniques attention directing-give, initial focus of attention-maintain, initial focus of attention-introducing, initial focus of attention-redirecting, infant response-no response, infant response-look, infant response-manipulate.

Coding system in which the stream of affective behavior of each partner is coded using 6 expressive modalities for the parent, which are vocalization, direction of gaze, head orientation, facial expression, body position, and specific handling of the infant and 5 for the infant which are vocalization, direction of gaze, head orientation, and facial expression.

Combination of expressive modalities, checked second by second, is transformed in one of the following seven adult monadic phases: avoid, avert, monitor, elicit, set, play, and talk. Six infant monadic phases are also coded: avoid, avert, monitor, set, play, and talk.

This coding scale assesses dyadic coregulation based on 5 patterns: symmetrical, asymmetrical, unilateral, disruptive, and unengaged. One additional pattern, no code, can be used for missing information.

This scale codes parent and infant positive and negative affective intensity in $30 \mathrm{~s}$ intervals. An ordinal 3-point scale (non, low, high) is used to code affective behaviors based on a combination of voice tone, facial expression,
Minde et al. [43] Minde et al. [42]

Jorg et al. [59] Schmücker et al. [58]

Landry [45] Landry [45]; Landry [49]

Tronick et al. [61] Feldman [74]

Feldman [60]

Fogel et al. [71] Sansavini [70]

Lunknenheimer et al. [71] 
TABLE 2: Continued.

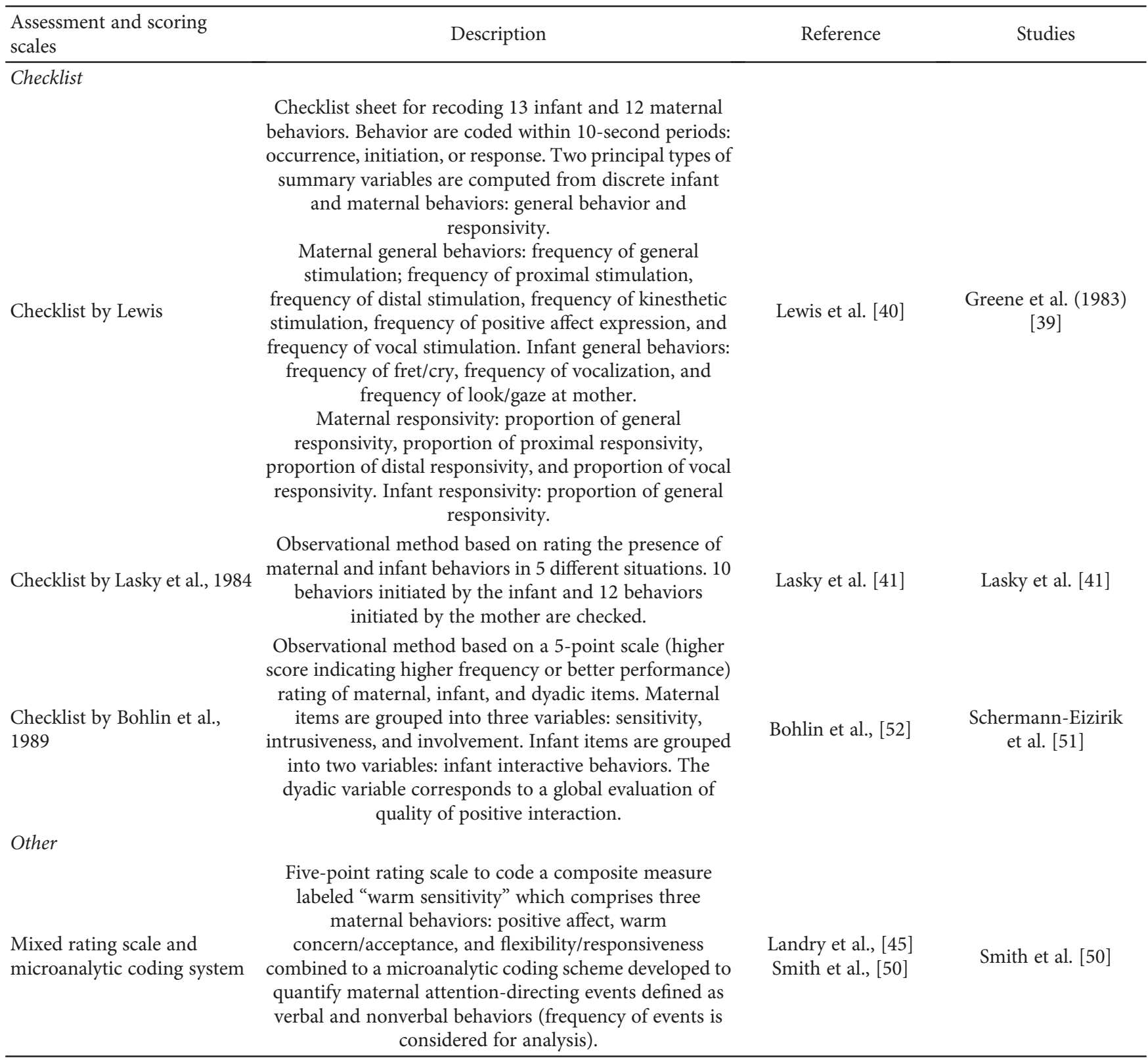

respectively, compared to controls. Interestingly, however, Schmücker and colleagues [58] also reported that preterm infants were more vocally active and responsive than fullterm infants were, thus indicating that the extent of responsiveness to the caregiver can be different depending on the communicative channel.

Two studies explored populations of full-term infants at high neurological risk, reporting abnormal infant behaviors within the parent-infant interaction [39, 51]. Specifically, Greene and colleagues [39] found that healthy infants looked significantly more at their mothers than sick infants, with sick full-term infants, corresponding to the group at highest neurological risk, having the lowest scores compared to healthy controls. Similarly, results by Schermann-Eizirik and colleagues [51] revealed that, unlike preterm born infants, full-term infants who required intensive care at birth, thus supposedly corresponding to the most impaired infants, differed from healthy full-term controls in their interactive patterns, with the first group showing significantly less interactive behaviors than the second one.

Finally, more recent studies investigating early motherinfant interactions within the first trimester of life showed no significant differences in terms of negative engagement or interactive patterns between preterm infants and fullterm controls [62, 67]. Only one study [69] reported more communicative behaviors in very low birth weight (VLBW) infants compared to full-term ones.

3.3.2. Parent (Mother) Interactive Behavior. Consistent with the results on infant behaviors during the first months of life, mothers of high-risk infants were described as less sensitive, 
more vigilant or intrusive, and less emotionally involved than mothers of healthy or low-risk infants.

At 3 months of age, Greene and colleagues [39] reported that, compared to healthy infants, high-risk infants, and specifically high-risk full-term infants, received more proximal and kinesthetic stimulation, but less distal and affective behaviors from their mothers during free play interactions. At the same infant age, other studies [62, 64, 67] reported that mothers of high-risk infants had more intrusive and less remote behaviors during face-to-face interaction than mothers of controls. Similarly, Minde and colleagues [42] reported that during feeding sessions ( 1 and 2 months) and play interactions ( 3 months), mothers of premature infants at higher risk provided more compensatory care (e.g., vocalization and face-to-face look), but less affect (e.g., smiling) to their infants compared to full-term mothers.

More inconsistent results were found about maternal sensitivity. In fact, while some studies $[53,58,67]$ failed to find differences between study and control mother groups in the sensitivity dimension, others showed that mothers of high-risk infants were less sensitive than mothers of controls were $[51,60]$. In particular, Schermann-Eizirik and colleagues [51] did not find differences in interactive behaviors between mothers of preterm infants, some of whom required intensive care, and mothers of healthy full-term infants. However, they observed significantly less sensitivity and less interactive involvement in mothers of high-risk full-term infants compared to mothers of healthy full-term infants at 4 and 6 months. Only one study reported enhanced sensitivity in mothers of high-risk infants, which was, however, associated with a higher level of intrusiveness [69].

Taken together, all these studies support the idea that mothers of high-risk infants are particularly focused on close monitoring and stimulating their infants rather than interacting with them in an affectionate or social manner.

3.3.3. Dyadic Interactive Patterns. Among the studies included in the current review, some approached a dyadic perspective, in addition to analyzing discrete maternal and infant dimensions, and focused on compromised patterns of synchrony [60], reciprocity [64], and positive exchanges within the dyad [51]. Two studies focused on the dyadic synchrony and reciprocity in the first semester of life and revealed that dyads at risk were less synchronized than control dyads were, showing less reciprocal rhythmic and fluent exchanges $[60,64]$. The study by Schermann-Eizirik and colleagues [51] revealed that high-risk dyads showed less positive exchanges compared to control dyads.

3.4. Comparison of Interactive Patterns between High-Risk and Non-High-Risk Dyads from 6 to 12 Months. Studies focusing on older infants evaluated more heterogeneous and difficult-to-compare interactive parameters, such as play, social, and communication skills. Consequently, also the results of these studies were more heterogeneous than were those observed during the first semester of life.

3.4.1. Infant Interactive Behavior. During toy-centered play sessions, both Muller-Nix et al. [55] and Landry et al. [45] found no significant differences between the study and control groups in infant play-interactive patterns, at 6 and 12 months, respectively. Conversely, significant differences in play-strategies between 12-month-old high-risk and control infants were found by Landry et al. and by Korja et al. $[49,65]$. Results by Landry and colleagues [49] revealed that high-risk infants showed, in general, less exploratory capacities compared to low-risk and healthy full-term infants and, more specifically, that high-risk infants were more dependent on mothers' structuring strategies than controls were. Similarly, Korja et al. [65] described 12-month-old preterm infants as less skilled in play, less attentive, and more apathetic, passive, and avoiding than controls during free play interactions.

A different approach was used by Farel and colleagues [46] who investigated interactive behaviors during feeding at 8 months of age and found that high-risk infants showed less clarity of cues and less responsiveness to their mothers than did controls. Finally, Smith and colleagues [50] found that high-risk infants had significantly lower expressive language abilities than controls during daily activity.

3.4.2. Parent (Mother) Interactive Behavior. As far as maternal behaviors are concerned, results of studies focusing on older infants highlighted two main altered dimensions. Consistent with studies focused on younger infants, mothers of high-risk older infants seemed to be more stimulating and less sensitive toward their infants than did mothers of control infants. For example, Landry et al. [45] found more attention-directing behaviors in high-risk mothers than in mothers of controls, while Farel and colleagues [46] found that mothers of high-risk infants reached lower scores in fostering cognitive growth behaviors than control mothers did.

Less affective behaviors were found in mothers of high-risk infants by Lasky et al. [41], Muller-Nix et al. [55], and Sansavini et al. [70]. Lasky and colleagues [41] reported that mothers of preterm infants restrain their infants less during a stressful clinical examination, maybe because they were more used to this kind of procedure. Muller-Nix et al. [55] found a negative gradient of maternal sensitivity, with mother of high-risk infants being less sensitive than mothers of low-risk infants that, in turn, were less sensitive than mothers of full-term infants. The study by Sansavini and colleagues [70], instead, revealed that mothers of extremely small for gestational age infants showed lower positive affect compared to mothers of full-term infants.

Finally, two studies [50,65] found no differences in maternal interactive behaviors between high-risk and control infant populations. However, both studies, differently from the other studies analyzing multiple discrete maternal dimensions, only reported a global score of maternal behaviors. Thus, whether significant differences would have been found, should single discrete maternal behaviors be analyzed, remains unanswered.

3.4.3. Dyadic Interactive Patterns. Among the studies focusing on dyadic interactive patterns at later infants' ages, Farel and colleagues [46] showed that high-risk dyads reached significantly worse total interactive scores, during feeding at 8 
months, compared to control dyads. Korja and colleagues [65] found no differences in dyadic mutuality, flatness, and disorganization and tension between 12-month-old highrisk and control infants. Finally, the study by Sansavini and colleagues [70] reported that extremely low gestational age (ELGA) dyads showed less frequent symmetrical coregulation and more frequent unilateral coregulation, specifically meaning that mothers observe, initiate, and demand doing something, while infants do not respond to them.

3.5. Coding Systems Used in Multiple Studies. In order to evaluate if similar patterns of mother-infant interaction could be inferred, we compared the quantitative results of those studies using the same coding systems. Only four scales were applied in more than one study (see Table 2).

The scale by Gunning et al. [68] and Murray et al. [15, 73] was used in two studies, in populations with the same characteristics and at the same age of assessment $[67,69]$. Consistent results were reported in "intrusiveness" and "remoteness" dimensions, describing mothers of high-risk infants as more intrusive and less remote than mothers of controls, while inconsistent results were reported for the "sensitivity" dimension.

The NCAST scale was also used in two studies $[46,53]$. The authors reported similar results in the feeding subscale, with a higher score in populations considered at high risk compared to control groups or normative data. However, investigated populations differed in clinical risk and age at the time of the mother-infant interaction assessment.

The CIB was used in other two studies $[62,64]$. They both found significant differences in the "intrusiveness" dimension, with mothers of the risk group reported to be more intrusive than mothers of the control group. However, the population characteristics of the two studies and the age at the time of the mother-infant interaction assessment differed between the two studies.

Finally, two studies by Landry et al. $[45,50]$ used the same coding system which was developed by the authors. Comparison of the data was however not feasible, as the results in one paper [45] were only related to mother behaviors, while the results of the other [49] were only related to infant behaviors.

No further quantitative comparisons, nor meta-analysis, were feasible with the selected articles, due to the heterogeneity of the publications and because the assessment tools and the dimensions analyzed were not consistent across the reports.

\section{Discussion}

In the last thirty years, extensive research has provided evidence that early interactive exchanges are fundamental in fostering later social and cognitive development, as they steadily drive, throughout early infancy and toddlerhood, emerging infant social abilities toward intentional and more complex relational capacities $[3-6,9,10,71]$. The occurrence of adverse perinatal events, however, negatively impacts the overall infant neurodevelopment with consequent detrimental effects also on infant social and relational dimensions $[7,8,11,15,17]$. The extent to which severe neonatal complications, such as the extremely preterm birth or low birth weight or the occurrence of neonatal brain insults, might affect early interactive exchanges between infants and their primary caregivers is, however, mostly uncharted. Our main objective was to review the studies that included the emerging behavioral interactive patterns of parent-infant dyads in infant populations at high neurological risk compared to control populations. We specifically focused on parent-infant interactions occurring over the first year of life, as it represents one of the most critical stages in infants' neurodevelopment and therefore is considered an optimal timeframe for early intervention on infants experiencing consequences of brain damage including developmental delays.

Most of the 18 studies resulting from our systematic search revealed that both infant and maternal behaviors within early interactions are compromised, which results, in turn, in a more general impairment of dyadic patterns. During the first six months of life, infants at high neurological risk are described as less engaged and active than control infants, which makes them less responsive social partners, unable to communicate cues that are sufficiently clear to their caregivers $[47,58,60]$. The most likely explanation of this finding is that these altered behaviors are primarily dependent on infants' neurophysiological immaturity and medical conditions, which necessarily affect their propensity to interact. This is consistent with the findings by Feldman [60] who evaluated neonatal biological rhythms and their relationship with mother-infant synchrony at 3 months of age. The author found that immature or dysregulated endogenous rhythms, due to perinatal events, limit the capacity of arousal modulation and negatively affect infant emotionality. During the same timeframe, i.e., the first semester of life, mothers of high-risk infants are more intrusive and overstimulating but, at the same time, less sensitive and affective $[51,60]$. Authors have generally interpreted these behaviors as the result of major maternal concerns relative to the health status of their infants. In this view, mothers tend to be more focused on infants' caretaking while lacking emotional involvement $[39,58]$.

It is of interest that the abnormalities observed in infant behavior tend to persist beyond the first semester of life, with infants being less engaging in the interaction and less focused during play sessions, while the intrusive maternal behaviors observed in early interactions evolve into more controlling and attention-directing behaviors in the second semester of life $[45,49]$. This has been interpreted as a process by which mothers become more conscious of their infants' compromised motor, cognitive, and communicative skills and shift from early concerns to the adoption of more suitable strategies to optimize their interactions with infants, such as directing their attention. Taken together, these results suggest that communicative patterns between mothers and infants at risk are less fluent and more disorganized compared to those typically observed in healthy populations and that the resulting quality of interaction is significantly impaired on the short and long terms.

Findings from the current review do not provide an exhaustive characterization of early mother-infant 
relationships in populations at very high risk of developing CP. In fact, many of the studies excluded infants with major neurological complications, thus leaving low birth weight and extreme prematurity as the primary selection criteria. While this selection approach limited the heterogeneity of the investigated samples, it clearly reduced the overall level of neurodevelopmental risk of the populations defined as at high risk. In spite of this important limitation, the available data support the concept that illness, rather than prematurity per se, gives the greatest contribution to the disruption of early infant interactive behaviors and, in turn, of maternal responses. In particular, our findings show that infants with more severe illness, either born prematurely or at term, have less optimal interactive approaches toward their mothers, as opposed to those with lower levels of neurodevelopmental risk [39, 41, 42, 45, 46, 50, 51, 58]. Similarly, maternal behaviors are directly related to infants' medical status with greater levels of infant risk associated with greater alterations of maternal interactive behaviors $[42,50,51,58]$. In general, mothers of infants who faced major neurological complications at birth were also more depressed, distressed, and anxious, as revealed by postnatal interviews or questionnaires, and these emotional states seem to influence mothers' interactive behaviors toward a less efficient perception of their infants' cues $[55,58,67,69]$. However, some inconsistency was found in relation to this aspect. Some other studies have found weak or no significant impact of maternal emotional states on the mother-infant interaction in high-risk populations, differently from the control population in which they seemed to play a bigger role $[42,53,58]$. Specifically, more negative emotional states were associated to poorer or more negatively affected maternal interactive patterns. Finally, few and discordant results were identified on the extent to which maternal emotional state compared to infants' risk can alter the interactive patterns $[55,58,64,69]$. Further investigations are therefore needed to disambiguate such aspects and, more importantly, to provide deeper insights on the maternal emotional state following the occurrence of perinatal adverse events and on the extent to which they can influence maternal interactive patterns over time.

It is of interest that the studies included in this work cover a time span of over thirty years. However, only older studies focused on very high neurological risk populations, while most recent ones mainly focused on prematurity. Two main considerations can be made. First, it is plausible that since first attempts at investigating early interactions in such complex populations have not been fully successful, subsequent attempts have mainly deviated toward more homogeneous populations including only premature infants. On the one hand, this approach has led to more consistent and reliable knowledge on early interactive exchanges in premature populations. On the other hand, however, this has also left many unanswered questions about the role of neurological illness in early mother-infant interactions. Second, the survival rate of infants at high risk for neurological impairments as indicated by current guidelines was significantly lower in the past decades, while it has significantly increased following advances in perinatal and neonatal care. Currently, more than ever, there is a critical need for the prompt referral of high-risk infants to diagnostic-specific early intervention, promoting early social interactions.

It is important to underline that findings were not consistent across studies. Inconsistent results were found in relation to maternal dimensions, in particular maternal sensitivity $[55,64,67,69]$, as well as to infant dimensions, in particular communicative patterns $[58,62,69]$. A number of factors might support these inconsistencies. Firstly, methods and scoring modalities used to investigate the interaction were very heterogeneous, varying from short video sessions to very long live observations and from microanalytic to global rating scoring systems. Different observational approaches and analyzed dimensions may result in heterogeneous pictures of dyadic exchanges. Secondly, studies were conducted at different infants' ages, albeit within the first year of life. Consequently, observational analyses were quite different across studies and specifically aimed at capturing the most appropriate interactive behaviors at different developmental stages. The last and the most important factor is that all articles included in this review focused on infant populations at high risk or neurologically impaired, but inclusion selection criteria were relatively variable, namely, varying from prematurity only to severe brain lesions. Therefore, not unexpectedly the extent of the interaction impairment was proportional and strictly related to the severity of infant medical risk.

Few studies have reported that early coping maternal behaviors influence later interaction maternal status [53]. Our results show that mothers of older infants demonstrate adaptive interaction strategies based on the impairment level of their infants. In particular, mothers of infants who clearly showed developmental delays as revealed by outcome measurements chose alternative strategies to properly communicate with their infants (i.e., g attention-directing gestures vs questioning) $[49,50]$. These findings might indicate a natural maternal attitude to adapt their behaviors based on infant needs over time $[45,49,53]$. Clearly, further investigations are needed in order to extend these results also in view of early interventions aimed at fostering such attitude as early as possible which would be of crucial importance.

In conclusion, results from this work extend previous research which has mainly focused on preterm populations, providing more information relative to early interactions involving infant population with or at high risk for neurological impairments. In fact, while our findings confirm that premature infants displayed behaviors similar to those previously observed in healthy populations, extremely preterm infants and full-term infants with severe illness showed markedly more impaired interactive patterns. Similarly, when maternal behaviors were taken into account, results showed that mothers of high-risk infants were more likely to show altered interactive patterns. However, while the studies reviewed here provided important information, the review did not yield a clear picture of early dyadic interactions in high-risk infant populations. Therefore, further investigation focusing on less heterogeneous populations (e.g., targeting infants with severe perinatal insults only versus controls) and embracing a longitudinal and comprehensive perspective, including, for instance, the systematic 
evaluation of maternal mental states and their impact on the interaction, are necessary to better characterize the extent to which early parent-infant interactions are impaired following severe perinatal insults. This is an essential step in order to determine the specific impact of addressing the promotion of positive parent-infant interactions as part of early intervention in infants at high neurological risk.

\section{Conflicts of Interest}

The authors declare that they have no conflicts of interest.

\section{Authors' Contributions}

Festante $\mathrm{F}$ and Antonelli $\mathrm{C}$ contributed equally to this work.

\section{Acknowledgments}

This review article was supported by the Italian Ministry of Health Grant RC 2018 to A. Guzzetta.

\section{References}

[1] U. Castiello, C. Becchio, S. Zoia et al., "Wired to be social: the ontogeny of human interaction," PLoS One, vol. 5, no. 10, article e13199, 2010.

[2] A. N. Meltzoff and M. K. Moore, "Imitation of facial and manual gestures by human neonates," Science, vol. 198, no. 4312, pp. 75-78, 1977.

[3] S. Braten, "Intersubjective enactment by virtue of altercentric participation supported by a mirror system in infant and adult," in Enacting Intersubjectivity: A Cognitive and Social Perspective on the Study of Interactions, vol. 10, pp. 133-147, IOS Press, Amsterdam, Netherlands, 2008.

[4] C. Trevarthen and K. J. Aitken, "Infant intersubjectivity: research, theory, and clinical applications," Journal of Child Psychology and Psychiatry, vol. 42, no. 1, pp. 3-48, 2001.

[5] D. N. Stern, "The interpersonal world of the infant," in A View from Psychoanalysis and Development Psychology, Basic Books, New York, NY, US, 1985.

[6] L. Murray, L. De Pascalis, L. Bozicevic, L. Hawkins, V. Sclafani, and P. F. Ferrari, "The functional architecture of motherinfant communication, and the development of infant social expressiveness in the first two months," Scientific Reports, vol. 6, no. 1, article 39019, 2016.

[7] M. Forcada-Guex, B. Pierrehumbert, A. Borghini, A. Moessinger, and C. Muller-Nix, "Early dyadic patterns of mother-infant interactions and outcomes of prematurity at 18 months," Pediatrics, vol. 118, no. 1, pp. e107-e114, 2006.

[8] R. C. White-Traut, K. M. Rankin, J. Yoder et al., "Relationship between mother-infant mutual dyadic responsiveness and premature infant development as measured by the Bayley III at 6 weeks corrected age," Early Human Development, vol. 121, pp. 21-26, 2018.

[9] M. H. Bornstein and C. S. Tamis-LeMonda, "Maternal responsiveness and cognitive development in children," New Directions for Child and Adolescent Development, vol. 1989, no. 43, pp. 49-61, 1989.

[10] R. Feldman and C. W. Greenbaum, "Affect regulation and synchrony in mother-infant play as precursors to the development of symbolic competence," Infant Mental Health Journal, vol. 18, no. 1, pp. 4-23, 1997.

[11] B. Beebe, F. Lachmann, S. Markese, and L. Bahrick, "On the origins of disorganized attachment and internal working models: paper I. A dyadic systems approach," Psychoanalytic Dialogues, vol. 22, no. 2, pp. 253-272, 2012.

[12] L. Giusti, L. Provenzi, and R. Montirosso, "The face-to-face still-face (FFSF) paradigm in clinical settings: socioemotional regulation assessment and parental support with infants with neurodevelopmental disabilities," Frontiers in Psychology, vol. 9, 2018.

[13] B. Beebe, D. Messinger, L. E. Bahrick, A. Margolis, K. A. Buck, and H. Chen, "A systems view of mother-infant face-to-face communication," Developmental Psychology, vol. 52, no. 4, pp. 556-571, 2016.

[14] E. M. Leerkes, A. N. Blankson, and M. O’Brien, “Differential effects of maternal sensitivity to infant distress and nondistress on social-emotional functioning," Child Development, vol. 80, no. 3, pp. 762-775, 2009.

[15] L. Murray, A. Fiori-Cowley, R. Hooper, and P. Cooper, "The impact of postnatal depression and associated adversity on early mother-infant interactions and later infant outcome," Child Development, vol. 67, no. 5, pp. 2512-2526, 1996.

[16] K. J. Aitken, "Intersubjectivity, affective neuroscience, and the neurobiology of autistic spectrum disorders: a systematic review," The Keio Journal of Medicine, vol. 57, no. 1, pp. 1536, 2008.

[17] R. Montirosso, C. Fedeli, L. Murray et al., "The role of negative maternal affective states and infant temperament in early interactions between infants with cleft lip and their mothers," Journal of Pediatric Psychology, vol. 37, no. 2, pp. 241-250, 2012.

[18] R. Korja, R. Latva, and L. Lehtonen, "The effects of preterm birth on mother-infant interaction and attachment during the infant's first two years," Journal of Pediatric Psychology, vol. 91, no. 2, pp. 164-173, 2012.

[19] M. Bozzette, "A review of research on premature infantmother interaction," Newborn and Infant Nursing Reviews, vol. 7, no. 1, pp. 49-55, 2007.

[20] World Health Organization (WHO), International Classification of Functioning, Disability and Health: ICF, World Health Organization, Geneva, Switzerland, 2nd edition, 2001.

[21] S. Grisaru-Granovsky, B. Reichman, L. Lerner-Geva et al., "Population-based trends in mortality and neonatal morbidities among singleton, very preterm, very low birth weight infants over 16 years," Early Human Development, vol. 90, no. 12, pp. 821-827, 2014.

[22] F. Serenius, K. Källén, M. Blennow et al., "Neurodevelopmental outcome in extremely preterm infants at 2.5 years after active perinatal care in Sweden," Journal of the American Medical Association, vol. 309, no. 17, pp. 1810-1820, 2013.

[23] P. S. Sutton and G. L. Darmstadt, "Preterm birth and neurodevelopment: a review of outcomes and recommendations for early identification and cost-effective interventions," Journal of Tropical Pediatrics, vol. 59, no. 4, pp. 258-265, 2013.

[24] L. W. Doyle, P. J. Anderson, M. Battin et al., "Long term follow up of high risk children: who, why and how?," BMC Pediatrics, vol. 14, no. 1, p. 279, 2014.

[25] M. K. Mwaniki, M. Atieno, J. E. Lawn, and C. R. J. C. Newton, "Long-term neurodevelopmental outcomes after intrauterine 
and neonatal insults: a systematic review," The Lancet, vol. 379, no. 9814, pp. 445-452, 2012.

[26] A. Pascal, P. Govaert, A. Oostra, G. Naulaers, E. Ortibus, and C. Van den Broeck, "Neurodevelopmental outcome in very preterm and very-low-birthweight infants born over the past decade: a meta-analytic review," Developmental Medicine \& Child Neurology, vol. 60, no. 4, pp. 342-355, 2018.

[27] I. T. Jarjour, "Neurodevelopmental outcome after extreme prematurity: a review of the literature," Pediatric Neurology, vol. 52, no. 2, pp. 143-152, 2015.

[28] E. Himpens, C. Van den Broeck, A. Oostra, P. Calders, and P. Vanhaesebrouck, "Prevalence, type, distribution, and severity of cerebral palsy in relation to gestational age: a meta-analytic review," Developmental Medicine \& Child Neurology, vol. 50, no. 5, pp. 334-340, 2008.

[29] A. Pappas and S. J. Korzeniewski, "Long-term cognitive outcomes of birth asphyxia and the contribution of identified perinatal asphyxia to cerebral palsy," Clinics in Perinatology, vol. 43, no. 3, pp. 559-572, 2016.

[30] E. E. Rogers and S. R. Hintz, "Early neurodevelopmental outcomes of extremely preterm infants," Seminars in Perinatology, vol. 40, no. 8, pp. 497-509, 2016.

[31] J. A. Eyre, M. Smith, L. Dabydeen et al., "Is hemiplegic cerebral palsy equivalent to amblyopia of the corticospinal system?," Annals of Neurology, vol. 62, no. 5, pp. 493-503, 2007.

[32] A.-C. Eliasson, L. Krumlinde-Sundholm, B. Rösblad et al., "The Manual Ability Classification System (MACS) for children with cerebral palsy: scale development and evidence of validity and reliability," Developmental Medicine \& Child Neurology, vol. 48, no. 7, p. 549, 2006.

[33] C. Morgan, I. Novak, R. C. Dale, A. Guzzetta, and N. Badawi, "GAME (Goals - Activity - Motor Enrichment): protocol of a single blind randomised controlled trial of motor training, parent education and environmental enrichment for infants at high risk of cerebral palsy," BMC Neurology, vol. 14, no. 1, p. 203, 2014.

[34] H. R. Rostami and R. A. Malamiri, "Effect of treatment environment on modified constraint-induced movement therapy results in children with spastic hemiplegic cerebral palsy: a randomized controlled trial," Disability and Rehabilitation, vol. 34, no. 1, pp. 40-44, 2011.

[35] I. Novak, C. Morgan, L. Adde et al., "Early, accurate diagnosis and early intervention in cerebral palsy: advances in diagnosis and treatment," JAMA Pediatrics, vol. 171, no. 9, pp. 897-907, 2017.

[36] M. Hadders-Algra, "Early diagnosis and early intervention in cerebral palsy," Frontiers in Neurology, vol. 5, 2014.

[37] C. Morgan, I. Novak, and N. Badawi, "Enriched environments and motor outcomes in cerebral palsy: systematic review and meta-analysis," Pediatrics, vol. 132, no. 3, pp. e735-e746, 2013.

[38] National Heart, Lung, and Blood Institute Website, "Development and use of quality assessment tools," https://www.nhlbi .nih.gov/health-topics/study-quality-assessment-tools.

[39] J. G. Greene, N. A. Fox, and M. Lewis, "The relationship between neonatal characteristics and three-month motherinfant interaction in high-risk infants," Child Development, vol. 54, no. 5, pp. 1286-1296, 1983.

[40] M. L. R. Lewis, The Effect of the Infant on Its Caregiver, Wiley-Interscience, Oxford, England, 1974.
[41] R. E. Lasky, J. E. Tyson, C. R. Rosenfeld, and N. F. Gant, "Maternal-infant interactions at one-year adjusted age in infants at low- and high-risk as newborns," Early Human Development, vol. 9, no. 2, pp. 145-152, 1984.

[42] K. Minde, M. Perrotta, and P. Marton, "Maternal caretaking and play with full-term and premature infants," Journal of Child Psychology and Psychiatry, vol. 26, no. 2, pp. 231244, 1985.

[43] K. K. Minde, P. Marton, D. Manning, and B. Hines, "Some determinants of mother-infant interaction in the premature nursery," Journal of the American Academy of Child Psychiatry, vol. 19, no. 1, pp. 1-21, 1980.

[44] K. K. Minde, M. Perrotta, and C. Corter, "The effect of neonatal complications in same-sexed premature twins on their mothers' preference," Journal of the American Academy of Child Psychiatry, vol. 21, no. 5, pp. 446-452, 1982.

[45] S. H. Landry, M. L. Chapieski, and M. Schmidt, "Effects of maternal attention-directing strategies on preterms' response to toys," Infant Behavior and Development, vol. 9, no. 3, pp. 257-269, 1986.

[46] A. M. Farel, V. A. Freeman, N. L. Keenan, and C. J. Huber, "Interaction between high-risk infants and their mothers: the NCAST as an assessment tool," Research in Nursing \& Health, vol. 14, no. 2, pp. 109-118, 1991.

[47] K. E. Barnard, M. A. Hammond, G. A. Sumner et al., "Helping parents with preterm infants: field test of a protocol," Early Child Development and Care, vol. 27, no. 2, pp. 255290, 1987.

[48] R. H. Bradley and B. M. Caldwell, "Home observation for measurement of the environment: a revision of the preschool scale," American Journal of Mental Deficiency, vol. 84, no. 3, pp. 235-244, 1979.

[49] S. H. Landry, P. W. Garner, S. Denson, P. R. Swank, and C. Baldwin, "Low birth weight (LBW) infants' exploratory behavior at 12 and 24 months: effects of intraventricular hemorrhage and mothers' attention directing behaviors," Research in Developmental Disabilities, vol. 14, no. 3, pp. 237-249, 1993.

[50] K. E. Smith, P. R. Swank, S. E. Denson, S. H. Landry, C. D. Baldwin, and S. Wildin, "The relation of medical risk and maternal stimulation with preterm infants' development of cognitive, language and daily living skills," Journal of Child Psychology and Psychiatry, vol. 37, no. 7, pp. 855-864, 1996.

[51] L. Schermann-Eizirik, B. Hagekull, G. Bohlin, K. Persson, and G. Sedin, "Interaction between mothers and infants born at risk during the first six months of corrected age," Acta Paediatrica, vol. 86, no. 8, pp. 864-872, 1997.

[52] G. Bohlin, B. Hagekull, M. Germer, K. Andersson, and L. Lindberg, "Avoidant and resistant reunion behaviors as predicted by maternal interactive behavior and infant temperament," Infant Behavior and Development, vol. 12, no. 1, pp. 105-117, 1989.

[53] L. Davis, H. Edwards, and H. Mohay, "Mother-infant interaction in premature infants at three months after nursery discharge," International Journal of Nursing Practice, vol. 9, no. 6, pp. 374-381, 2003.

[54] H. I. McCubbin, M. A. McCubbin, J. M. Patterson, A. E. Cauble, L. R. Wilson, and W. Warwick, "CHIP. Coping Health Inventory for Parents: an assessment of parental coping patterns in the care of the chronically ill child," Journal of Marriage and the Family, vol. 45, no. 2, p. 359, 1983. 
[55] C. Muller-Nix, M. Forcada-Guex, B. Pierrehumbert, L. Jaunin, A. Borghini, and F. Ansermet, "Prematurity, maternal stress and mother-child interactions," Early Human Development, vol. 79, no. 2, pp. 145-158, 2004.

[56] A. P. Scheiner and M. E. Sexton, "Prediction of developmental outcome using a perinatal risk inventory," Pediatrics, vol. 88, no. 6, pp. 1135-1143, 1991.

[57] P. Crittenden, CARE-Index: Coding Manual, Unpublished Manuscript, Miami, FL, USA, 1979-2004, http://www. patcrittenden.com.

[58] G. Schmücker, K. H. Brisch, B. Köhntop et al., "The influence of prematurity, maternal anxiety, and infants' neurobiological risk on mother-infant interactions," Infant Mental Health Journal, vol. 26, no. 5, pp. 423-441, 2005.

[59] M. Jorg, R. Dinter, F. Rose et al., "A system of categories for the assessment of early mother-child interaction," Zeitschrift fur Kinder- und Jugendpsychiatrie, vol. 22, no. 2, pp. 97106, 1994

[60] R. Feldman, "From biological rhythms to social rhythms: physiological precursors of mother-infant synchrony," Developmental Psychology, vol. 42, no. 1, pp. 175-188, 2006.

[61] E. Tronick, H. Als, and T. B. Brazelton, Monadic Phases: a Structural Descriptive Analysis of Infant-Mother Face to Face Interaction, Merrill Palmer Quarterly, 1980.

[62] R. Feldman and A. I. Eidelman, "Neonatal state organization, neuromaturation, mother-infant interaction, and cognitive development in small-for-gestational-age premature infants," Pediatrics, vol. 118, no. 3, pp. e869-e878, 2006.

[63] R. Feldman, Coding Interactive Behavior (CIB) Manual, Unpublished manual, Bar Ilan University, Ramat-Gan, Israel, 1998.

[64] R. Feldman, "Maternal versus child risk and the development of parent-child and family relationships in five high-risk populations," Development and Psychopathology, vol. 19, no. 2, pp. 293-312, 2007.

[65] R. Korja, J. Maunu, J. Kirjavainen et al., "Mother-infant interaction is influenced by the amount of holding in preterm infants," Early Human Development, vol. 84, no. 4, pp. 257267, 2008.

[66] R. Clark, "The parent-child early relational assessment: instrument and manual," in Madison: University of Wisconsin Medical School, Department of Psychiatry, 1985.

[67] F. Agostini, E. Neri, S. Dellabartola, A. Biasini, and F. Monti, "Early interactive behaviours in preterm infants and their mothers: influences of maternal depressive symptomatology and neonatal birth weight," Infant Behavior and Development, vol. 37, no. 1, pp. 86-93, 2014.

[68] M. Gunning, A. Fiori-Cowley, and L. Murray, The Global Ratings of Mother-Infant Interaction Scoring Manual, 2nd edn, Winnicott Research Unit, University of Reading, Reading, England, 1999.

[69] E. Neri, F. Agostini, P. Salvatori, A. Biasini, and F. Monti, "Mother-preterm infant interactions at 3 months of corrected age: influence of maternal depression, anxiety and neonatal birth weight," Frontiers in Psychology, vol. 6, 2015.

[70] A. Sansavini, V. Zavagli, A. Guarini, S. Savini, R. Alessandroni, and G. Faldella, "Dyadic co-regulation, affective intensity and infant's development at 12 months: a comparison among extremely preterm and full-term dyads," Infant Behavior and Development, vol. 40, pp. 29-40, 2015.
[71] E. S. Lunkenheimer, S. L. Olson, T. Hollenstein, A. J. Sameroff, and C. Winter, "Dyadic flexibility and positive affect in parentchild coregulation and the development of child behavior problems," Development and Psychopathology, vol. 23, no. 2, pp. 577-591, 2011.

[72] A. Fogel, I. de Koeyer, C. Secrist, A. Sipherd, T. Hafen, and M. Fricke, The Revised Relational Coding System, 2003, Department of Psychology, University of Utah, Unpublished manuscript.

[73] L. Murray, C. Stanley, R. Hooper, F. King, and A. Fiori-Cowley, "The role of infant factors in postnatal depression and mother-infant interactions," Developmental medicine and child neurology, vol. 38, no. 2, pp. 109-119, 1996.

[74] R. Feldman, "Infant-mother and infant-father synchrony: the coregulation of positive arousal," Infant Mental Health Journal, vol. 24, no. 1, pp. 1-23, 2003. 


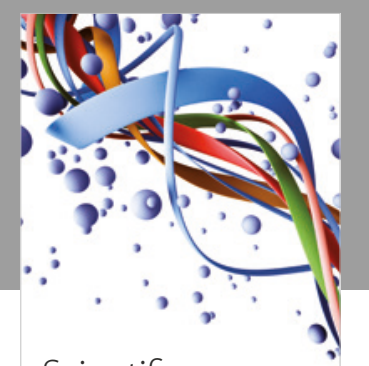

Scientifica
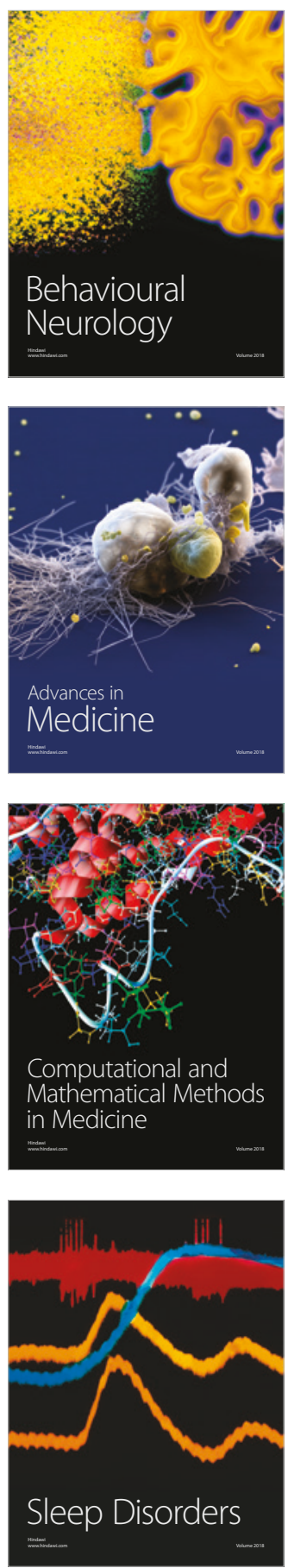

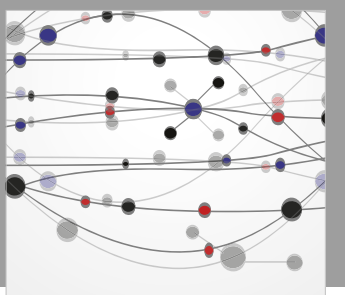

The Scientific World Journal

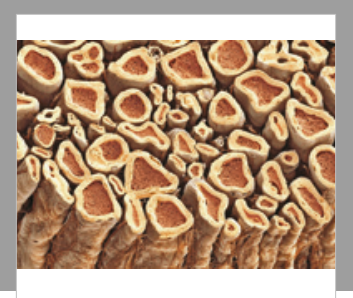

Case Reports in

Neurological Medicine

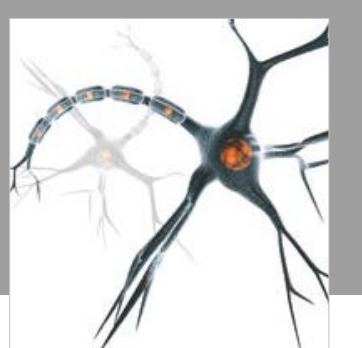

Neural Plasticity

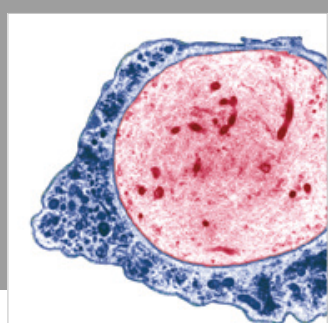

Multiple Sclerosis

International

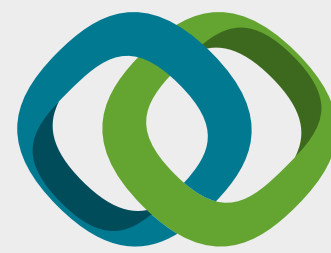

Hindawi

Submit your manuscripts at

www.hindawi.com
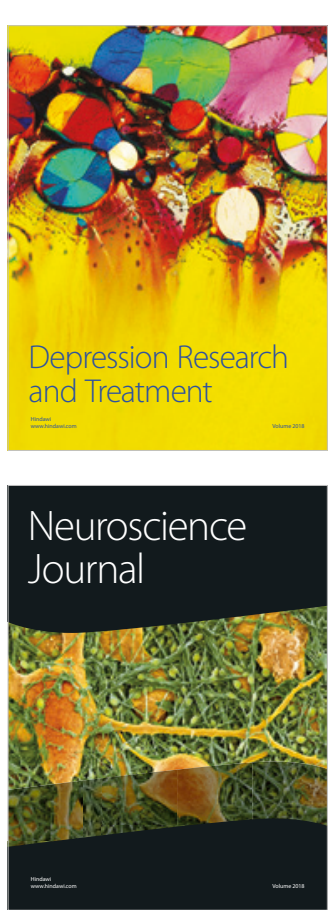

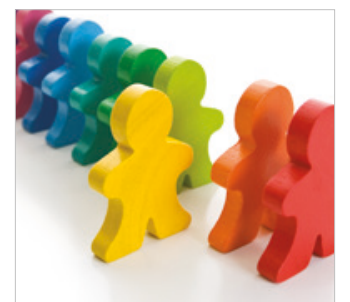

Autism

Research and Treatment
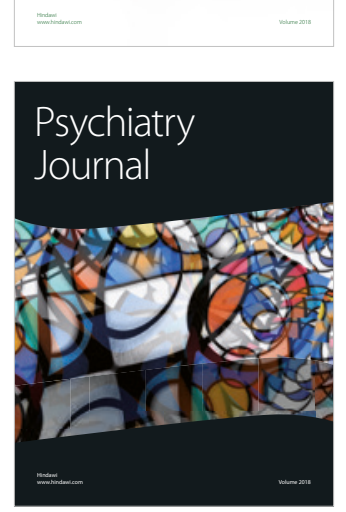
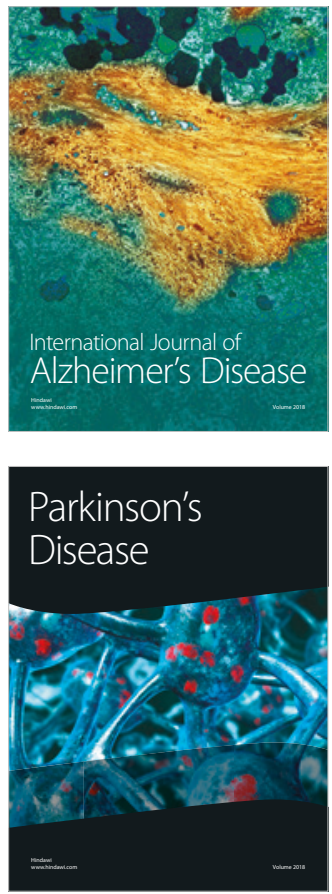
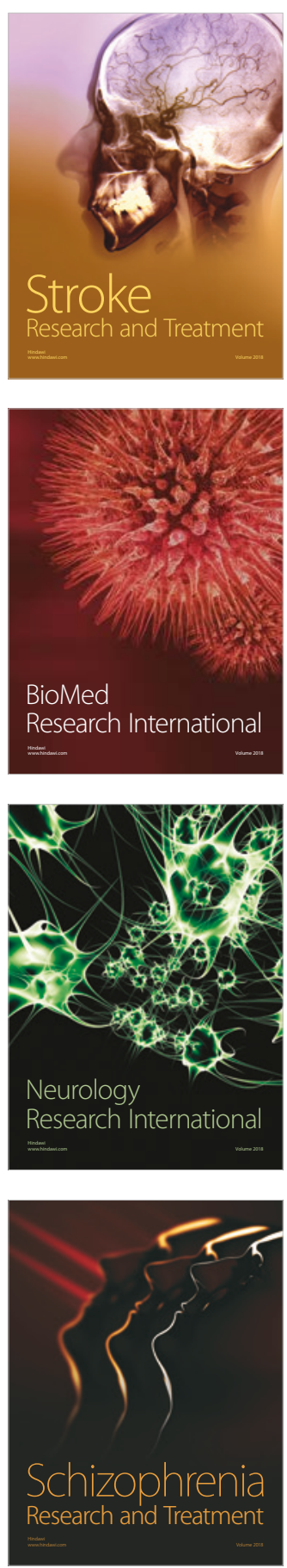